\title{
In-Silico Evaluation of an Iterative Pace-Mapping Technique to Guide Catheter Ablation of Ventricular Ectopy
}

\author{
Andony Arrieula ${ }^{1,2,3}$, Hubert Cochet $^{2,4,5,6}$, Pierre Jaïs ${ }^{2,4,5,6}$, \\ Michel Haïssaguerre ${ }^{2,4,5,6}$, Mark Potse $^{3,2,1}$ \\ ${ }^{1}$ CARMEN Research Team, Inria Bordeaux Sud-Ouest, Talence, France \\ ${ }^{2}$ IHU Liryc, fondation Bordeaux Université, Pessac, France \\ ${ }^{3}$ Univ. Bordeaux, IMB, UMR 5251, Talence, France \\ ${ }^{4}$ Univ. Bordeaux, CRCTB, U1045, Bordeaux, France \\ ${ }^{5}$ INSERM, CRCTB, U1045, Bordeaux, France \\ ${ }^{6}$ Bordeaux University Hospital (CHU), Electrophysiology and Ablation Unit, Pessac, France
}

\begin{abstract}
Ventricular tachychardia (VT) is one of the mechanisms that induce sudden cardiac death. A way to cure VT is to ablate the exit site with an endocardial catheter. But it can be difficult, and sometimes impossible, to localize this exit site accurately enough.

We propose a process to accelerate catheter ablation using an automatic method to guide the catheter towards the exit site. The proposed process uses the QRS complex integral of the 12-lead ECG. The method was tested using a realistic numerical forward model with pacing sites in endocardial, epicardial, and mid-myocardial tissue.

With 12 pacing sites, 6 targets had been approximated to less than $1 \mathrm{~mm}$. Five more were within $5 \mathrm{~mm}$ distance, and one was at $10 \mathrm{~mm}$ distance.

We conclude that although the convergence is sometimes erratic, the proposed method does converge to the exit site, often within the radius of an ablation lesion.
\end{abstract}

\section{Introduction}

Catheter ablation is a curative treatment for drugresistant ventricular tachycardia (VT) and ectopic beats. The site(s) to ablate are usually found by first mapping the activation pattern of the arrhythmia with the help of an electro-anatomical mapping system. But this method is time-consuming and requires that the arrhythmia can be induced in the catheterization laboratory, and be sustained by the patient for several minutes. Many of the most lifethreatening VTs do not meet this criterion and are considered "unmappable".

The purpose of this study is to test a method based on repeated ventricular pacing to guide a catheter iteratively to the site of origin, using nothing but the pacing-induced 12-lead ECGs and the ECG of a single ectopic beat or VT cycle.

The method is based on the assumption that, if a ventricular beat has a single site of origin, there is an approximately linear relation between the coordinates of the origin and the QRS integral of the ECG. This simple approximation has recently been shown to hold well in clinical [1] as well as in numerical tests [2], but the localization errors are still too large to direct ablation without further confirmation. However, when this method is used with information from pacing sites near the origin it may be expected to be more accurate $[3,4]$. Therefore we tested an iterative method which uses repeated predictions and corrections to converge to the site of origin.

The method works as follows: first a single ectopic beat or VT cycle is captured. Then the ventricles are paced at four locations separated by several centimeters and not located on a line and the paced ECGs are captured. In each ECG the signals are integrated over the QRS interval. Using the four paced beats a linear relation between the ECG vector and the site of origin is estimated. This relation is applied to the ectopic ECG to provide a first prediction of the site of origin. Then the ventricles are paced at this location, the ECG is captured, and the linear relation is reestimated with all five pacing locations. This is repeated until the predicted location appears to converge. A flow diagram of this method is shown in figure 1 . The method is complicated by the fact that the predicted location may be outside the myocardium, deep in the wall, or otherwise not reachable by a catheter.

We estimated if, and how fast, this method converges to a location that is sufficiently close for ablation. Tests were performed in a realistic numerical model, for subendocardial, intramural, and subepicardial sites of origin. 


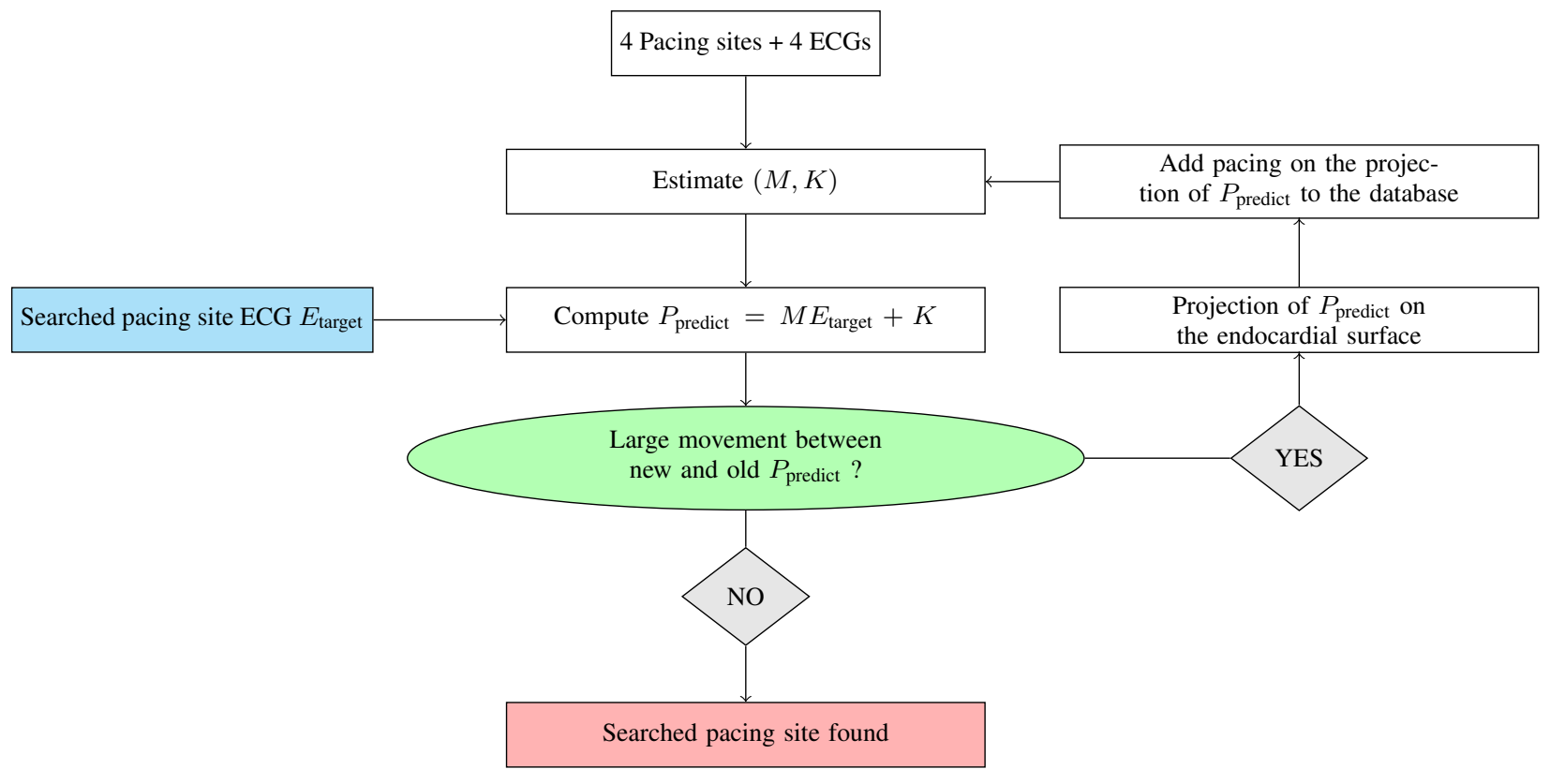

Figure 1. Process to find location of unknown pacing site

\section{Methods}

The method that we tested is based on the assumption that, for a ventricular beat originating from a single point $P$ the QRS integral of the $N$-lead ECG $(E)$ is linearly related to $P$ :

$$
P=M E+K
$$

where $M$ is a $3 \times N$ matrix and $K$ a 3 -element vector. The elements of $M$ and $K$ are fitted using a set of known $P$ and corresponding $E$.

To test the method we used a detailed anatomical model of the heart and torso previously created from computed tomography data [2] of a single patient (figure 2). A total of 12 arrhythmia exit sites $\left(P_{\text {target }}\right)$ was chosen randomly in the ventricular model such that 2 were subendocardial, 2 intramural, and 2 subepicardial in each ventricle. For each site the ventricular activation and 12-lead ECG were simulated with stimulation at $P_{\text {target }}$.

For an initial estimation of $M$ and $K$ we stimulated the ventricles at 4 different sites to cover the widest possible area of the ventricle. For left-ventricular (LV) targets we used LV pacing sites, and for right-ventricular (RV) targets only RV pacing sites. For each pacing site the ventricular activation and ECG were simulated. The parameters $M$ and $K$ were estimated by solving the linear system consisting of equation 1 repeated for each of these 4 sites, with known $P$ and $E$, in a least-squares sense.

Then, for each target site, the following procedure was followed. Using the estimated $(M, K)$ we made a first prediction $P_{\text {predict }}$ of each arrhythmia exit site using equa-

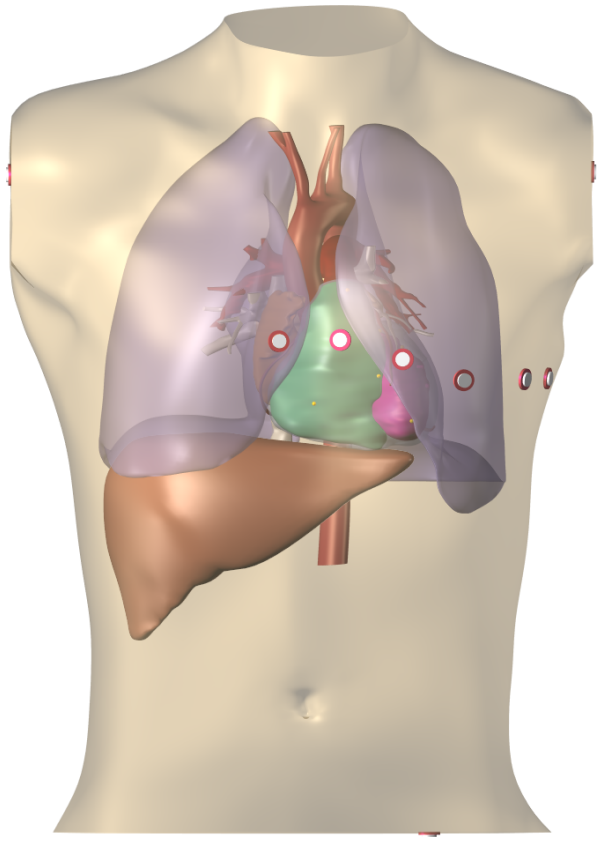

Figure 2. Heart-torso model used for the ECG simulations, with the 9 electrodes of the standard 12-lead ECG. The limb electrodes were placed on the cut surfaces, to approximate a placement on the actual limbs, which were not available in the imaging data. 
tion 1. Assuming that pacing is only possible on the endocardium, the next pacing site $P_{\text {pace }}$ was chosen as the projection of $P_{\text {predict }}$ on the endocardium. The ventricles were then stimulated at $P_{\text {pace }}$ and ECGs were again computed. The resulting pair $\left(P_{\text {pace }}, E_{\text {pace }}\right)$ was then added to the pace-mapping database, and $(M, K)$ were re-estimated to compute the next pacing site.

Since $P_{\text {predict }}$ was not guaranteed to lie inside the myocardium or even near the heart, its projection on the endocardium was achieved with an exhaustive search on all endocardial nodes in the 3-dimensional computational mesh, which had a uniform edge length of $0.2 \mathrm{~mm}$.

Propagating action potentials were simulated with a monodomain reaction-diffusion model using Ten Tusscher-Panfilov 2006 membrane dynamics [5]. ECGs were computed using a lead-field method [6]. The simulations were performed with a recent version of the Propag-5 software $[6,7]$ and were run on a cluster computer.

\section{Results}

Figure 3 shows the euclidian distance between the estimated point $P_{\text {predict }}$ at each step of the process and the target point $P_{\text {target }}$.

The results show a wide variation in convergence. With 12 pacing sites, 6 of the 12 targets had been approximated to an acceptable distance of $1 \mathrm{~mm}$, including all 4 endocardial points. Five more were within $5 \mathrm{~mm}$ distance, and a last was at approximately $10 \mathrm{~mm}$ distance.

We found a better convergence for $\mathrm{LV}$ targets than for $\mathrm{RV}$ targets $(1.85 \mathrm{~mm}$ average at the end for the $\mathrm{LV}$, $3.06 \mathrm{~mm}$ for the RV).

\section{Discussion}

Using computer simulations on a realistic human model we tested a proposed iterative pace-mapping method that could be applied in the catheterization laboratory to guide an ablation catheter to the exit site of an unmappable arrhythmia. In this test the proposed method identified the exit site with reasonable accuracy in $90 \%$ of cases. All endocardial target sites were localized with less than $1 \mathrm{~mm}$ accuracy. Epicardial and intramural sites were associated with larger errors. This may be explained by the fact that pacing could only be done on the subendocardium.

For one of our target sites the algorithm converged poorly $(>10 \mathrm{~mm})$. This site was located in the RV. We hypothesize that the smaller contribution of the RV to the ECG reduces the strength of the linear relation between $P$ and $E$. The more complex shape of the RV may also play a role.

We used only LV information to localize LV sites and only RV information to localize RV sites. This was done

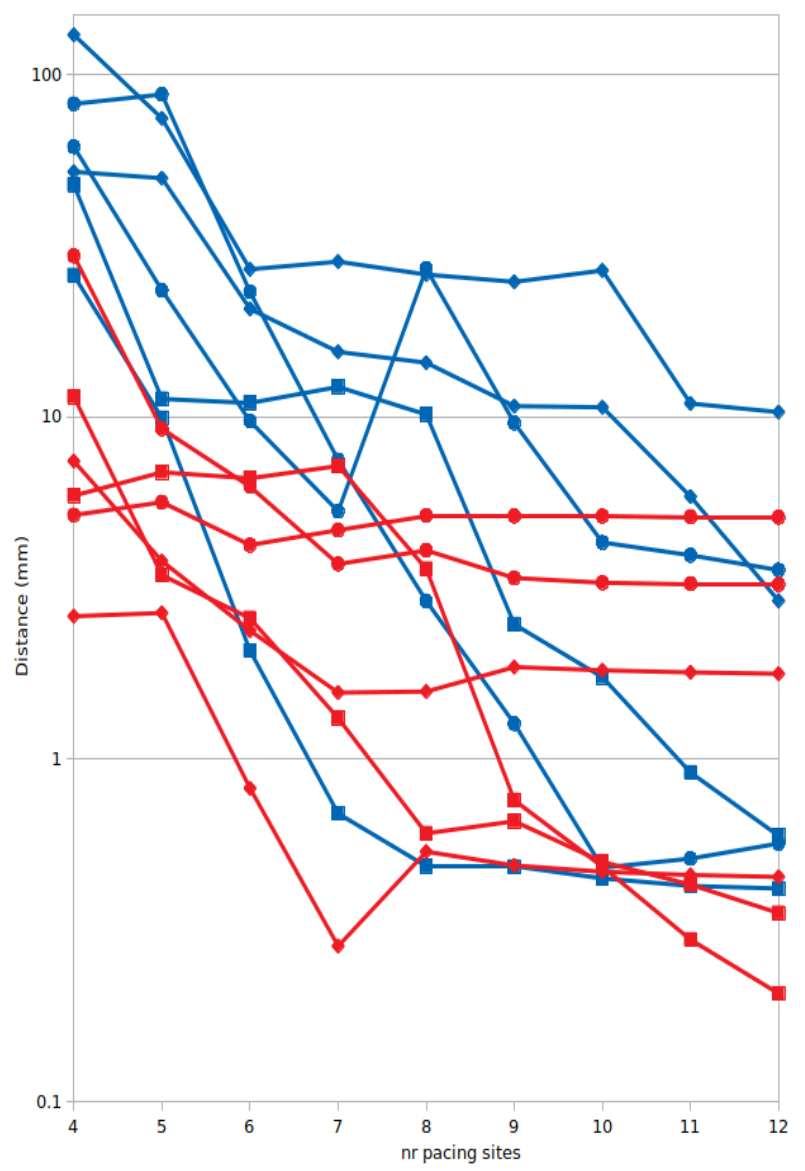

Figure 3. Euclidian distance between estimated point and target point at each iteration. The red curves represent left-ventricular targets, blue curves right-ventricular targets. Squares represent endocardial targets, diamonds intramural targets, and circles epicardial targets. Note that the vertical scale is logarithmic to capture the large range.

to mimic the most common clinical cases, where the electrophysiologist is likely to correctly guess the ventricle of origin based only on the ECG of the arrhythmia. If the arrhythmia is thought to originate in the RV, a (more difficult) LV access can be avoided, but mapping is then obviously restricted to the RV. For supposedly LV origins this restriction is not so pertinent, because usually during LV mapping an RV catheter will be present as well. We did not yet assess the effect of pacing sites from both ventricles on the performance of the procedure.

For this study we used only the signals from the 9 electrodes that are used for a standard 12-lead ECG. More general inverse electrocardiographic models use much larger lead sets. Initial work on simple interpolation methods such as ours was performed with a set of 64 electrodes [3]. However, recent work has shown that the added benefit 
of more than 9 electrodes is very small [2] and tests on clinical data have also shown good results with a 12-lead ECG [1].

In the current implementation we added only pacing sites that were as close as possible to the estimated exit sites. This "hasty" method is not necessarily the most efficient in all cases. It may be better to advise the cardiologist to first pace three points around the estimated location, to obtain a region-specific $M$. In this case, it would also be relevant to remove more distant sites from the database. It may also be possible to infer which information is missing in $M$ and to select the next pacing site such as to maximize the relevant information it provides. The limitation most relevant for a clinical equivalent is that we did not remove database points that were apposed more closely than the measurement accuracy. This could lead to large errors when the measured locations are inaccurate.

Another limitation of the current study is that it was performed on a single patient-specific model, with normal wall thickness and no scar. More extensive testing is required before a clinical test can be envisaged.

\section{Conclusion}

We tested an iterative pace-mapping algorithm based on a simple linear model to predict the site of origin of a ventricular arrhythmia from the QRS integral of the 12-lead ECG. The algorithm found the site of origin with sufficient accuracy for ablation in $90 \%$ of cases, and performed less well for intramural and epicardial sites, which an endocardial catheter cannot reach.

\section{Acknowledgements}

This work was supported by the Région NouvelleAquitaine, grant nr. 2017 - 1R50109 - 00013434; the European Research Council (H2020 grant agreement number 715093, ECSTATIC); and the French National Research Agency, grant references ANR-10-IAHU04-LIRYC and ANR-11-EQPX-0030. This work was granted access to HPC resources of CINES under GENCI allocation 2019A0050307379.

\section{References}

[1] Sapp JL, Bar-Tal M, Howes AJ, Toma JE, El-Damaty A, Warren JW, McInnis PJ, Zhou S, Horáček BM. Real-time localization of ventricular tachycardia origin from the 12-Lead electrocardiogram. JACC Clin Electrophysiol 2017;3:687699.

[2] Kania M, Coudière Y, Cochet H, Jaïs P, Potse M. Prediction of the exit site of ventricular tachycardia based on different ECG lead systems. In Pickett C, Corsi C, Laguna P, MacLeod R (eds.), Computing in Cardiology, volume 44. Rennes, France, 2017; 253.

[3] Potse M, Linnenbank AC, Peeters HAP, SippensGroenewegen A, Grimbergen CA. Continuous localization of cardiac activation sites using a database of multichannel ECG recordings. IEEE Trans Biomed Eng May 2000;47(5):682689.

[4] Latour A, Grimbergen CA, Peeters HAP, Linnenbank AC, Sippens Groenewegen A. Quantitative algorithm for the localization of the origin of ectopic ventricular beats using catheter pace mapping. In Proc. 18th Annu. Int. Conf. IEEE EMBS. 1996; .

[5] ten Tusscher KHWJ, Panfilov AV. Alternans and spiral breakup in a human ventricular tissue model 2006; 291:H1088-H1100.

[6] Potse M. Scalable and accurate ECG simulation for reactiondiffusion models of the human heart. Front Physiol 2018; 9:370.

[7] Krause D, Potse M, Dickopf T, Krause R, Auricchio A, Prinzen FW. Hybrid parallelization of a large-scale heart model. In Keller R, Kramer D, Weiss JP (eds.), Facing the Multicore-Challenge II, volume 7174 of Lecture Notes in Computer Science. Berlin: Springer, 2012; 120-132.

Address for correspondence:

Andony Arrieula

CARMEN research team

Inria Bordeaux Sud-Ouest

200 Avenue de la vieille tour

33450 Talence, France

andony.arrieula@inria.fr 